\begin{abstract}
ARTICULO
Revista Derecho - Año 3 edicion 507 - 20

Web: http://revistas.unap.edu.pe/rd E-mail: revistaderecho@unap.edu.pe

ISSN 2313-6944

\section{BIOETICA Y DERECHO. ¿ES POSIBLE SU EFICACIA?}

Boris Espezúa Salmón ${ }^{*}$

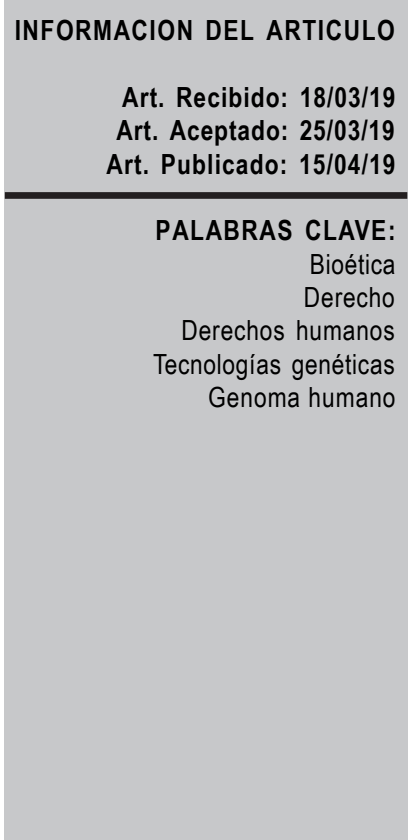

\section{RESUMEN}

En las últimas décadas, la bioética ha cobrado inusitada importancia, por el crecimiento de la tecnología, los riesgos de la medicina y por lo tanto de la vida misma, lo que amerita tener que recurrir al derecho para regular sus implicancias. Por su parte, las tecnologías biotecnológicas resultan ambivalentes, pues su uso es susceptible de generar grandes beneficios para la humanidad, pero también grandes riesgos derivables de un mal uso cuyas consecuencias son imprevisibles y que pueden afectar no sólo a quienes las emplean sino al resto de la humanidad, e incluso a las generaciones futuras. Ante ello el Derecho deberá establecer los límites de lo permitido; de ahí deriva la estrecha relación entre Bioética y Derecho, entendido como norma de conducta que emana de la voluntad de todos. Entender los valores constitucionales y los «principios generales de las naciones civilizadas» como acuerdo mínimo: a la luz de la Declaración de Derechos Humanos y de las demás declaraciones internacionales y convenios que forman parte de nuestro mundo, es el desafío que tiene esta comunión de Bioética y Derecho, que debe asegurar su eficacia en tiempos que la vida en su significado más amplio se encuentra amenazada.
\end{abstract}

Docente principal de la Facultad de Ciencias Jurídicas y Políticas de la Universidad Nacional del Altiplano de Puno.

espezualmon@hotmail.com 


\section{BIOETICS AND RIGHT. IS YOUR EFFECTIVENESS POSSIBLE?}

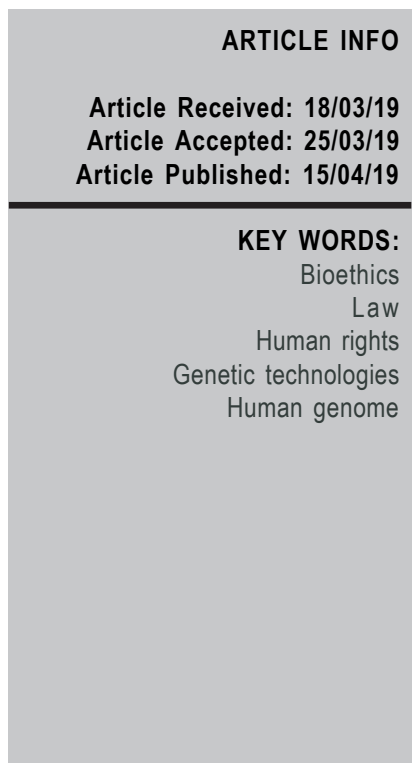

\section{ABSTRACT}

In recent decades, bioethics has become unusually important, due to the growth of technology, the risks of medicine and therefore of life itself, which merits having to resort to the right to regulate its implications. On the other hand, biotechnological technologies are ambivalent, since their use is likely to generate great benefits for humanity, but also great risks derived from misuse whose consequences are unpredictable and that can affect not only those who use them but also the rest of them. humanity, and even future generations. Given this, the Law must establish the limits of what is allowed; Hence the close relationship between Bioethics and Law, understood as a norm of behavior that emanates from the will of all. Understanding the constitutional values, and the «general principles of civilized nations» as a minimum agreement: in the light of the Declaration of Human Rights and the other international declarations and conventions that are part of our world, it is the challenge that this communion of Bioethics and Law, which must ensure its effectiveness in times that life in its broadest meaning is threatened. 


\section{INTRODUCCION}

La Bioética ha devenido en una disciplina inter y multidisciplinar. Su comprensión como su aportación suscita una reflexión transversal, es concebida como una rama fundamental y en ella participan disciplinas diversas para aportar sus conocimientos, tiene que ver con darle sentido teleológico al trabajo tecnológico que involucra la vida humana, y la vida en general, y nos brinda la construcción conjunta de pautas que nos permitan tratar los problemas que las biotecnologías nos plantean. Y que a todos nos conciernen en tanto que seres humanos, individualmente y colectivamente en tanto que constituimos una sociedad interrelacionada y plural, que comparte un espacio territorial. En las últimas décadas la biología y la medicina han avanzado más que otra ciencia. Sus descubrimientos, sus resignificaciones, sus experimentos, producen mayor conmoción a la humanidad y suscitan mayores interrogantes que el hallazgo del fuego en su momento. Corresponde precisamente a las actuales generaciones establecer una oportuna respuesta y regulación legal y política basado en un consenso que vaya más allá de escuelas y creencias. Por ello, éste involucramiento tiene dos niveles: El nivel político y el nivel jurídico. Por el primero es el pueblo en su situación de poder soberano quien decide lo más conveniente a su futuro, a las mejoras de su actual situación. Para ello, tiene que convocar el representante genuino del pueblo expresado que es el gobernante para que los ciudadanos puedan manifestarse ya sea por la vía del referéndum o por la vía del sufragio. El nivel jurídico es que también la justicia es soberana y exige que el pueblo también se pronuncie a través de sus legisladores que son elegidos por el pueblo, es decir en ese sentido es la extensión de su expresión y representación, por lo que tienen toda la legitimidad para poder emanar leyes que tenga que controlar, encauzar, poner límites a los trabajos biotecnológicos que tengan que ver con la salud y la vida humana.

En los años setenta, las relaciones entre seres humanos, ciencias y medicina tuvieron cambios importantes ya que creció en el progreso biotecnológico y ello supuso la oportunidad de intervenir en los aspectos vitales esenciales. En este contexto surgió la Bioética como disciplina con enfoque plural, encaminadas a conseguir el bien social. V. Potter, señala que: la Bioética es «el conocimiento de cómo usar el conocimiento», el puente entre ciencias y humanidades. En este escenario no es difícil constatar, que las materias tratadas por la Bioética requieren la intervención del mundo jurídico, que se vuelque en el interés práctico de quienes laboran en la Bioética práctica cuyas nuevas implicaciones y responsabilidades no resultan menos complicadas.

Revista Derecho - 5 (2019) 9 
Casado (2000) sostiene que los problemas de la Bioética deben ser debatidos por la sociedad en su conjunto antes de que sean adoptadas soluciones normativas, sobre las que, en una sociedad democrática y plural, es preciso lograr consenso. Un consenso que, en cuestiones que atañen a los valores individuales y colectivos en forma tan especial, resulta difícil conseguir. Por ello, conviene centrar la búsqueda del compromiso en la elaboración de unas reglas del juego aceptables para la mayoría de los ciudadanos independientemente de sus opciones ideológicas. Se trata de problemas que, al no tener una respuesta social unívoca, desembocan en una demanda de legislación y eso deviene una típica cuestión de axiología jurídica: cuáles son los valores que debemos proteger y cómo debe hacerse. Ante la posibilidad de intervenir en los procesos biológicos hay discrepancias sociales que generan conflictos, y son éstos los que requieren de la intervención del Derecho para establecer los límites a la libertad de actuación individual. El Derecho deberá establecer los límites de lo permitido; de ahí deriva la estrecha relación entre la Bioética y el Derecho -entendido como norma de conducta que emana de la voluntad de todos.

Aquí, se debe tomar en cuenta que hay que ver al Derecho como la disciplina que conjunciona tanto el elemento moral como el elemento positivizable, es decir como el dualismo jurídico ha querido establecer con Gregorio Peces Barba que superaba al dicotomía de que el derecho desde el enfoque positivista ortodoxo no concebía como elemento compositivo a la moral, a lo cual los jusnaturalistas pugnaban en reprochar y establecer que en la construcción de nuestras normas legales, a moral era fundamental para darle derrotero, finalidad a las normas jurídicas. Superado esta discusión que caracterizó la primera centuria del siglo XX, Tanto los Neopositivistas como los neo-jusnaturalistas aceptan ahora la composición de la norma jurídica tanto de moral como de validación estatal.

Las normas jurídicas poseen una evidente relación con las morales, aunque no dependan de la ética para su configuración, y a lo largo de los siglos el análisis de estas relaciones ha sido una cuestión central del pensamiento filosófico-jurídico. En nuestro tiempo vivimos el auge del pluralismo moral de la sociedad y que es un valor constitucionalmente protegido.

\section{1.- Materiales y Métodos.}

En el presente trabajo se tomará en cuenta el método Cualitativo, que permitirá realizar análisis e interpretación de textos, poniendo en algunos casos como ejemplos casos o hechos vinculados a la Bioética y Derecho. Se utilizará las fichas de observación como instrumentos. No se considera muestreo,

10 Revista Derecho - 5 (2019) 
población, ni universo, porque es un artículo que rebasa el territorio nacional y regional.

\section{2. ¿Cuál es la relación entre Bioética y Derecho?}

La Bioética tiene su base en cuatro principios morales: autonomía, beneficencia, no maleficencia y justicia distributiva. A través de ellos, se distinguen las alternativas y para la resolución de los problemas e iniciar un debate sobre éstos. En base a estos principios bioéticos, se funda la Ley General de Salud de 1982, cuyo objeto es constituir las condiciones de atención de las personas en nuestro país. El problema de la Bioética es descubrir la solución a los dilemas médico-legales. Tealdi (2008) precisa al respecto que un dilema que implica un conflicto de decisión entre dos o más opiniones, con diferente interpretación moral y con otro resultado. Los dilemas se incrementan, y uno de ellos es el significado de la vida, identificando su inicio y su fin. Este dilema tiene varias implicaciones jurídicas. Citando al gran jurisconsulto Marco Tulio
Cicerón: «La ciencia que se aparte de la justicia más que ciencia debe llamarse astucia». Actualmente la Bioética es una recurrencia muy crítica y a la vez emplazativa y esta interactuando con el derecho. La biotecnología, entiende que el Derecho se ubicará en un plano de igualdad para disipar los conflictos que se engendren con motivo del progreso científico. Lo que se quiere es que entre la Bioética y el Derecho alcancen la protección del ser humano y su hábitat, así como proteger la raza humana y su entorno orgánico.

Lo que conlleva a un análisis ético y que toma en cuenta principios como el respeto a la integridad corporal, el respeto a la capacidad de ejercer como persona el reconocimiento de la diversidad y la equidad, tanto de género como de grupos sociales actual situación, sumados a ello los problemas del aborto, la muerte asistida, el tema de la manipulación del $\mathrm{ADN}$, las células madres que nos coloca en un incómodo trinomio de compromiso: persona, dignidad, vida, lo que supone un problema inicial para la Bioética ${ }^{1}$. Es importante debatir su sen-

\footnotetext{
${ }^{1}$ Concretamente en España, están reguladas las técnicas de reproducción asistida, la donación y utilización de fetos y embriones, la utilización, liberación y comercialización de organismos modificados genéticamente con el fin de prevenir los riesgos para la salud humana y el medio ambiente, y ciertas conductas referidas a la manipulación genética se sancionan en el código penal. Además, la Unión Europea ha elaborado numerosas directivas sobre organismos modificados genéticamente, riesgos laborales, protección de datos, patentes, etc. También los organismos internacionales han forjado textos como el Convenio sobre Diversidad Biológica de Río de Janeiro, auspiciado por las Naciones Unidas, o la Declaración de los Derechos de las Generaciones Futuras y la Declaración Universal sobre el Genoma Humano, de la UNESCO, que pretenden completar la Declaración Universal de Derechos Humanos. Y, como se ha repetido anteriormente, contamos con el Convenio para la Protección de los Derechos Humanos y la Dignidad del Ser Humano con respecto a las aplicaciones de la Biología y la Medicina, elaborado por el Consejo de Europa como organismo específicamente encargado de su protección y promoción de los Derechos Humanos en el ámbito europeo, pero abierto a la firma de cuantos países lo deseen.
} 
tido y divulgar sus fines ante los ciudadanos. Las nuevas tecnologías genéticas están ocasionando cambios de gran impacto social, moral y jurídico, por lo que constituyen posibilidades para comprender actitudes ambivalentes que requieren de la realización de un debate social informado y del establecimiento de normas éticas, sociales y jurídicas. Dichas nuevas tecnologías genéticas reclaman investigadores, médicos, humanistas, políticos, informadores. La racionalidad en la discusión reclama una información previa, que no es la pura razón instrumental, mucho menos, sino una razón estimativa, que tome en cuenta el sentido humano y moral

Todo ello plantea consenso en la ética. ¿Existe o no desacuerdo en los principios morales? En todo caso ¿pueden coexistir visiones diversas con respecto a la ética vinculado a la vida? La discusión entre las posiciones universalistas y las que hacen hincapié en la diversidad y el pluralismo debe plasmarse en marcos de tolerancia, comprensión del «otro» que nos lleven a ponernos en la situación de nuestros semejantes. El principal problema estriba en que la ética en consenso no tiene respuestas a los problemas de la bioética mientras que, por el contrario, por ejemplo la teología sí tiene respuestas dogmáticas que cuentan con el aval de siglos de utilización de la fe sin ocasionar fisuras entre sus creyentes. Lo cual nos lleva de nuevo a los Derechos Huma- nos y a la posibilidad de acuerdos, al interés por el bien común, que resulta un poco difícil en sociedades absorbidas por el individualismo, sobre los que hay que trabajar para asegurar reconocimiento y protección concreta.

Dichos Derechos Humanos pretenden proteger la vida, la libertad, la igualdad, la seguridad, la integridad y la dignidad de un ser humano, por lo que se propone que deben ser respetados por todas las personas. Ello implica que los individuos asuman responsabilidades a partir de la ampliación de sus espacios de libertad pero reconociendo los espacios de los otros.

En general, por ejemplo en las aplicaciones biomédicas de las nuevas tecnologías no se suele plantear el problema de ser ilícitas sino más bien de cuáles son los límites a su licitud. Ante ellas. El Derecho se debate entre una actitud no intervencionista para el investigador y una postura controladora, que emerge cuando la preocupación social así lo aconseja. Una mera información inconsistente obtenida del tema del Genoma Humano por ejemplo puede afectar a derechos como la intimidad, la no-discriminación, la autonomía, el pluralismo, la dignidad de la persona o a la libertad de investigación. Eduardo Tinant (2012) señala que la bioética normativa trata de orientar los avances de la tecnociencia y los cambios sociales y culturales de la globalización, re- 
curriendo a los derechos humanos, a los valores y principios éticos universalmente aceptados. Por consiguiente, en esa lógica entendemos que el Derecho no tiene como función detener el desarrollo de la ciencia y las nuevas tecnologías, sino que se va a encargar de orientarlo, controlarlo y regularlo. El Derecho, como proceso de interacción entre valores, norma y conducta humana intersubjetiva, tiene como fin supremo la defensa de la persona y el respeto de su dignidad, como reza el artículo primero de la Constitución Política del Perú, por lo que no puede exponerse aquellas acciones que potencialmente puedan ser lesivas a la especie humana en forma integral.

Consecuentemente, el Derecho debe estar atento a los cambios científicos tratando de controlar los riesgos potenciales de los mismos, sin ser un obstáculo a la innovación, sino actuando como elemento de racionalización y de garantía. Sin perder de vista la necesidad de que sus normas estén dotadas de la temporalidad limitada que requiere una materia en continua evolución y basadas en el consenso que, idealmente, debe seguir al debate social informado que tanto se preconiza, además porque debe cumplir su rol de control, de regulación y de garantizar la adecuada vida de los seres humanos y de la naturaleza que nos rodea.

\section{3.- ¿Es posible el amalgamiento interdisciplinar y actuar eficazmente?}

Se debe insistir en la necesidad de establecer una regulación que prevenga y frene los posibles abusos. Pero, como se ha indicado más arriba, dicha normativa ya existe tanto en el ámbito nacional como internacional; lo que ocurre es que la mera existencia de normas es insuficiente. Regular desde arriba no basta; es necesaria una información rigurosa y un debate previo sobre las implicaciones de la biotecnología que permita llegar a establecer acuerdos, aunque sean parciales y revisables, pero, la importancia de dichos acuerdos, deben tener el carácter de legitimados, sólo así se podrá avanzar democráticamente para que los fines de la bioética así como del Derecho tengan en su amalgamiento una función constructiva y humana. Los principios de la bioética pretenden responder a estas preguntas ¿Quién debe decidir? ¿Qué daño o beneficio se puede causar? ¿Cómo se debe tratar a un individuo en relación con los demás? ¿Qué se debe decir desde el Derecho? ¿Cómo garantizamos la protección de la dignidad? Las respuestas a estas preguntas requieren un alto encaminamiento de la justicia.

Méndez (2004) sostiene que los seres humanos somos «por naturaleza» culturales -es decir, adaptadores del medio- y está en nuestras manos elegir y actuar moralmente. El respeto y pro-

Revista Derecho - 5 (2019) 13 
moción de los Derechos Humanos son la mejor pauta a la hora de juzgar la bondad y maldad de las biotecnologías y las prácticas biomédicas. Ellos representan el consenso logrado en torno a cuáles son las exigencias de la dignidad humana. A ello se le llama, también Bioética Jurídica, que es uno de los campos de trabajo de esta nueva disciplina en relación a la bioética normativa, la cual se encarga de la regulación, de los temasproblema que enfrenta. La bioética jurídica da cuenta de una bioética reguladora a partir de las decisiones tomadas colectivamente para aplicarlas en el tratamiento de nuevos conflictos bioéticos.

La libertad y la salud son valores muy próximos. Hoy en día son casi idénticos. El derecho a la vida del sujeto, ocupa un lugar principal en las declaraciones de los derechos humanos en Europa, Estados Unidos y América Latina, juegan un papel importante: el derecho a la salud pasa a ser entendido socialmente, bioéticamente y legalmente como un correlato del derecho a la vida. En ese sentido los artículos 2 y 3 de la Convención Europea de Derechos Humanos se refieren al derecho a la vida y al derecho a respetar la integridad física de la persona, como por ejemplo el derecho a no padecer tortura, trato degradante y castigo inhumano, o analiza en el campo médico la mala praxis, el error, la negligencia o la imprudencia, razones de deterioro de la salud de los pacientes. $\mathrm{Al}$ respecto la Bíoética cumple una función redimitiva que tiene que ver con proteger, evitar y remediar los abusos, las arbitrariedades del poder económico, político o de otra índole, como de regular la negligencia, o el actuar doloso u omisivo de los particulares. Otro aspecto importante del nexo entre el derecho y la medicina en el tratamiento médico en ocasiones como del «trato degradante» al paciente. Todo ello, constituyen criterios legales que protegen toda clase de intervenciones médicas y el tratamiento está definido en la observación del principio de buena fe. La información es una parte esencial de este principio y un elemento precioso en el equilibrio entre la buena fe y el trato degradante. Pero la interpretación, cuanto al equilibrio correcto y sostenido, no sólo se expresa en términos jurídicos, sino éticos, principistas, filosóficos

Los significados jurídicos determinan también nuestro concepto público de salud. Es una correcta cultura de legalidad que nos hace respetar lo estatuído, lo normado. Esto queda evidenciado en los procedimientos de consentimiento informado. El dar información y el seguimiento de este servicio es una obligación para la ley en todos los contextos clínicos. La atención sanitaria solo será legalmente correcta mientras el sujeto reciba la información correcta: tanto en contenido cuanto por el procedimiento adecuado. El cumplimiento jurídico implica la capacidad de la persona para recibir la información, con sujeción al procedimiento correcto más que en el contenido, visto desde la 
perspectiva de un acto de buena fe, es decir, que nos encontramos con una instancia de la aplicación de un principio legal.

La bioética y el derecho, es un campo fusionado de estudio que tiene por objeto a uno mayor: el derecho biomédico. En tanto que parte de la bioética, tiene que ver con la Medicina, Biología, Psicología, el método que se sigue en ese campo no es el propio de la ciencia del derecho, sino tal vez el de la filosofía. En este sentido, el proceder en bioética y derecho está especialmente cercano al de la disciplina filosófica de la ética. Ambas materias tratan filosóficamente un objeto jurídico. El estatus disciplinar ambiguo del conocimiento que se obtiene en el campo de conocimiento denominado bioética y derecho no es algo que resulte hoy tan extraño, cuando hablamos de un mundo interconectado. La eficacia de ambos es el resultado de una construcción paulatina que ha entrado actualmente, en la época de la revolución tecnocientífica. En ese sentido, la multidisciplinariedad que se predica de la bioética -y que es otra forma de aludir a su naturaleza filosófica- no resulta hoy para nada algo excepcional. Lo que sí. Hay que destacar es que la misión de la bioética no consiste en absoluto en sentar unos principios inamovibles que, a modo de nuevo derecho natural biológico, vinculen de forma absoluta a la sociedad y al legislador democrático. La misión de la bioética, por el contrario, consiste más bien en la información, la aclaración conceptual y el análisis de los avances en biomedicina con dimensión ética. Su objetivo es iluminar a las instancias que tienen la función de decidir sobre los mismos. Está en contra del monopolio exclusivo en la interpretación de los valores -que es lo que durante un tiempo hicieron las Iglesias respecto a los estados confesionales- y que ahora convertirían a la bioética y a sus expertos en un peligro para la democracia. La bioética jurídica es como una filosofía comprometida con los problemas de su tiempo. Los avances en biomedicina exigen decisiones democráticas, esas decisiones exigen, por su parte, razones, que inevitablemente remiten a justificaciones, a conceptos y a valores que nacen de la discusión plural y abierta propia de las sociedades que respetan la libertad.

\section{4.- ¿Cómo interviene el Derecho?}

Anna Arendt (2003) ha señalado que, si los avances en biomedicina fueron el combustible principal del tren bioético, y si puede decirse asimismo que la filosofía puso las vías por las que circuló dicho tren, el Derecho ha sido el maquinista que lo ha conducido hasta donde se encuentra ahora. Una de las causas principales de esa cierta preeminencia del derecho en el seno de la bioética. Es innegable que los avances biomédicos que poseen una dimensión ética poseen muchas veces, también, una dimensión jurídica. Afectan en mu- 
chos casos a cuestiones sociales fundamentales, como el sistema de parentesco, los usos del cuerpo, el tiempo del nacimiento y de la muerte, cuestiones que ya venían reguladas jurídicamente y que, tras esos avances, resulta necesario volver a regular. Pensemos por ejemplo en la técnica de los trasplantes de órganos, la cual exige decidir sobre el momento de determinación de la muerte, o bien en las técnicas de reproducción asistida, cuya implantación trastoca las normas jurídicas vigentes sobre la filiación. Ahora bien, hay dos posiciones en la relación de la Bioética y el Derecho, la que defiende la tesis de la separación tajante entre la bioética y el derecho y la que defiende la inclusión del discurso jurídico en el seno de la bioética, pueden señalarse aquí como las más extendidas para la organización de dichas relaciones. Los que defienden la tesis de la separación tajante entre la bioética y el derecho suelen partir del postulado que afirma que la bioética tiene un carácter intrínsecamente ético y normativo. Este carácter suyo es lo que le permite prefigurar al derecho. La bioética fundamental sirve para fundar los principios básicos que deben ser respetados, principios de los cuales las normas jurídicas pueden ser deducidas. La bioética entonces, y según esto, sirve para señalar el mínimo ético básico que la legislación debe siempre respetar. Atribuye así esta postura a la bioética el papel de dirección de la legislación, y al derecho el papel de brazo armado de la bioética. La relación que, de acuerdo con esta postura, se establece entre la bioética y el derecho es estrecha, pero permite separar a la vez con nitidez ambas instancias, a las que atribuye funciones claramente diferenciadas. De acuerdo con la tesis de la inclusión del discurso jurídico en el seno de la bioética, significa de una parte que la bioética, a la que podemos denominar bioética y derecho, que es la que se ocupa, especialmente, del entrelazamiento de los aspectos éticos y jurídicos de las actividades biomédicas. Bioética y derecho, por lo tanto, no es más que el nombre para una fracción más del amplio movimiento bioético: la fracción que estudia los problemas de la interacción entre la sociedad y la biomedicina en tanto que dichas actividades con dimensión ética son, además, objeto de decisiones.

La especial importancia que tienen para la bioética los aspectos jurídicos reside en que los verdaderos principios de la bioética, los cuales permiten resolver todas las cuestiones planteadas, son los derechos fundamentales proclamados en las constituciones de los Estados. Lo cierto es que ni los problemas éticos son inexistentes en una sociedad pluralista, ni los derechos fundamentales pueden servir para dar solución a todos los problemas planteados por los avances de la biomedicina. La estudiosa Siverino (2010) señala que hay problemas bioéticos para cuya solución, tales derechos constituyen efectivamente una referencia válida. Pero hay otros problemas para cuya solución la apela-

16 Revista Derecho-5 (2019) 
ción a tales derechos resulta ambigua o inconcluyente. Es lo que pasa, por ejemplo, en las discusiones sobre el aborto y la eutanasia, las cuales implican muchas veces, precisamente, la cuestión acerca de quién es el titular de esos derechos fundamentales. Por último, hay asuntos donde la apelación a los derechos fundamentales resulta del todo inútil. Un buen ejemplo de uno de ellos es el que se refiere a la investigación con animales. Los derechos de los animales, ya son una realidad en muchos países, lo que va creando mayor conciencia de su reconocimiento y protección. Es por todo ello por lo que, y sin necesidad de aceptar que el campo de estudio llamado bioética y derecho constituya la parte central de la bioética, podamos afirmar aquí que integra una parte muy importante de la misma, es decir es implícito, sobreentendido. Una parte que se ocupa de la dimensión normativa de las actividades de la biomedicina en tanto que éstas tienen repercusión jurídica. En las sociedades actuales, estas normas jurídicas tienen poco que ver con el respeto a unas creencias morales homogéneas en toda la sociedad, el pluralismo va ganando terreno, de tal manera que el gran despegue de la ciencia y la tecnología acarrean una serie de problemas éticos, y es que no todo lo técnicamente posible es, necesariamente, éticamente recomendable. Este es uno de los postulados esenciales de la Bioética, disciplina que tiene más de cuarenta años de existencia y que se ocupa de las relaciones entre la ética, equidad, desarrollo, tecnología y sociedad y que tiene como todo nuevo enfoque adherentes y detractores. Lo ideal que por una salud general, es que todos estemos a favor de su consideración y aplicación.

La tarea del Derecho es proteger las estructuras profundas de la sociedad, apoyar el orden público y mantener el Estado de Derecho, sus criterios y sus principios. Haberle (2004) sostiene que: la legislación que se refiere al campo de la medicina, como por ejemplo en litigios, en el área contenciosa o bien en cuestiones de seguros, de responsabilidad, o la protección de la propiedad intelectual, en ningún momento ha fallado en su papel. Los procesos de juridicidad y de interiorización del conocimiento jurídico se desarrollan sin trabas. ¿Cómo justifican entonces los abogados no haber percibido los cambios producidos en los discursos interconectados con el derecho? Esta no es una cuestión meramente retórica. Los juristas han elaborado una teoría para dar respuesta a estas preguntas. Esta teoría trata, en su parte esencial, de cómo el derecho puede reglamentar todos los aspectos de la vida social en todas las circunstancias. La presuposición de tal poder organizativo implica el principio tal vez más escondido en el derecho, de que la realidad social es de por sí, siempre y sin excepción, objeto de calificación jurídica. Por tanto, no hay realidad médica que no pueda ser objeto de calificación jurídica. Esta apertu- 
ra garantiza tanto una interpretación jurídica omnipresente como una calificación de la realidad. Las virtudes de la democracia moderna consideran la juridificación y la medicalización como un bien común. La definición del bien común presupone que cada uno de nosotros tiene derecho a la atención médica o jurídica. Estas medidas solo se pueden dar bajo las condiciones epistemológicas que han sido descritas. El espíritu de este Derecho es nuestro concepto de la justicia.

\section{5.- Conclusiones.}

En nuestro país, mediante el Decreto Supremo No 011-2011-JUS publicado el 27 de julio del 2011 en el diario oficial «El Peruano», se aprobaron los «Lineamientos para garantizar el ejercicio de la Bioética desde el reconocimiento de los Derechos Humanos», en los cuales se destaca: (i) el reconocimiento integral del valor y sentido de la vida humana, y el respeto de la dignidad humana como fin y no como medio, señalándose además que estos son límites inamovibles para todos aquellos sujetos que emplean las ciencias, cuya meta es la investigación e intervención en personas; y, (ii) la posibilidad de que toda persona pueda beneficiarse del progreso de las ciencias y tecnologías en un marco de respeto de su dignidad y demás derechos fundamentales.

Luego de la adopción de la Declaración Universal sobre Bioética y
Derechos Humanos, y antes de la entrada en vigencia del Decreto Supremo $\mathrm{N}^{\circ}$ 011-2011-JUS, se presentaron decisiones jurisprudenciales en las cuales se mencionan expresamente algunos de los principios de la bioética mencionados anteriormente en este artículo. Tales son los casos de N.N. contra el Ministerio Público sobre cambio de identidad y nombre, y el de Miguel Ángel Morales Denegri sobre el derecho a rechazar tratamiento médico.

La ciencia ha llegado a colisionar con sus características inherentes: dignidad y libertad. Dicho ello, y siendo que el abordar esta temática implica cuestiones técnicas muy complejas y especialmente sensibles para la sociedad, es ineludible un estudio interdisciplinario que nos brinde un marco conceptual que permita analizar el impacto de las nuevas tecnologías en el ser humano, en su entorno y ambiente. Es posible, por ejemplo, que la biotecnología intervenga desde momentos iniciales en la configuración misma del ser humano, en una etapa en la que no tiene capacidad de elección -a través de la eugenesia o la predeterminación genética de los individuos-; asimismo, se realizan simultáneamente, en diferentes países, proyectos de investigación o experimentaciones médicas, se importan y exportan células madres embrionarias, órganos y tejidos, se mantienen en vida a seres humanos con medidas de soporte vital a pesar de su falta de consentimiento. 
Tal como sostiene Tinat (Ibidém) es dable apreciar deberes éticos negativos, correlativos a prohibiciones jurídicas, y deberes positivos (de promoción o de virtud), correlativos a obligaciones jurídicas de dar y de hacer o no hacer, así como considerar al derecho positivo el resultado de los sistemas de valores que coexisten en una sociedad. Es en este escenario que la bioética jurídica, aporta una lectura distinta de las categorías jurídicas: resultando ser la herramienta idónea para el análisis y resolución de casos que nos permitirá elevarnos y realizarnos mejor, como seres humanos.

\section{Bibliografía}

ARENDT Hanna. (2003) «Responsabilidad y juicio» Edit. Trotta. Barcelona- España.

CASADO GONZÁLES. María. (comp.) (2000) Estudios de Bioética y Derecho. Valencia- España. Editorial Tirant lo Blanch.

HABERLE Peter. (2004) «Nueve ensayos constitucionales y una lección jubilar» Edic. Palestra Lima- Perú.

MÉNDEZ BAIGES Víctor. (2004) «El Filósofo y el mercader» (FCE) junto con Héctor Claudio Silveira Gorski, Bioètica y Derecho (Editorial UOC.
SIVERINO BAVIO, Paula. (2010) «Bioética jurídica y derechos fundamentales: breve propuesta en relación al estudio del derecho de las personas». En: Revista de Derecho de Familia y de las Personas. Año 2, número 9. Buenos Aires: La Ley.

TEALDI, Juan Carlos. (2008) Bioética de los Derechos Humanos. Investigaciones Biomédicas y Dignidad Humana. México, D.F.: Universidad Nacional Autónoma de México.

TINANT, Luis Eduardo. (2012) «Principios Jurídicos, Principios Bioéticos» Revista de Derecho PUCP N. 69. LimaPerú..

UNESCO- PRENSA. (2015) «La Declaración Universal sobre Bioética y Derechos Humanos adoptada por la Conferencia General de la UNESCO». Consulta: 10 de julio del 2015. Disponible en <http:// portal.unesco.org/es/ $\mathrm{e} \mathrm{v} \cdot \mathrm{p} \mathrm{h} \mathrm{p} \mathrm{-} \mathrm{U} \mathrm{R} \mathrm{L}$ $=30274 \& U R L \_D O=D O$ - T O P I C \& U R L _ SECTION $=201 . \mathrm{html}>$ 
20 Revista Derecho - 5 (2019) 